\title{
ON THE FUNDAMENTAL REGIONS OF A FIXED POINT FREE CONSERVATIVE HÉNON MAP
}

\author{
MÁRIO BESSA and JORGE ROCHA
}

\author{
(Received 16 April 2007)
}

\begin{abstract}
It is well known that an orientation-preserving homeomorphism of the plane without fixed points has trivial dynamics; that is, its non-wandering set is empty and all the orbits diverge to infinity. However, orbits can diverge to infinity in many different ways (or not) giving rise to fundamental regions of divergence. Such a map is topologically equivalent to a plane translation if and only if it has only one fundamental region. We consider the conservative, orientation-preserving and fixed point free Hénon map and prove that it has only one fundamental region of divergence. Actually, we prove that there exists an area-preserving homeomorphism of the plane that conjugates this Hénon map to a translation.
\end{abstract}

2000 Mathematics subject classification: 54H20, 37B99, 28D05.

Keywords and phrases: free maps of the plane, Hénon map, area-preserving maps, topological conjugacy, fundamental regions.

\section{Introduction and statement of the results}

Given a metric space $X$, two homeomorphisms $f, g$ of $X$ are topologically conjugated if there exists a homeomorphism $h$ such that $g \circ h=h \circ f$ (the map $h$ is called a topological conjugacy). That is, $g$ can be obtained from $f$ by a $C^{0}$ change of coordinates $\left(g=h \circ f \circ h^{-1}\right)$ which in particular implies that these maps have the same topological dynamical properties (for example, existence of fixed/periodic points, homoclinic points and non-wandering points).

In this paper we consider an area-preserving map derived from well-known Hénon map defined by $H_{a}(x, y)=\left(a-x^{2}-y, x\right)$, and we prove that, in the area-preserving setting and for $a<-1$, this map is topologically conjugated to a translation. More precisely the main result is as follows.

THEOREM 1. There exists an area-preserving topological conjugacy between the conservative Hénon map and $T(x, y)=(x+1, y)$.

The first author was supported by FCT-FSE, SFRH/BPD/20890/2004. The second author was partially supported by FCT-POCTI/MAT/61237/2004.

(C) 2008 Australian Mathematical Society 0004-9727/08 \$A2.00+0.00 


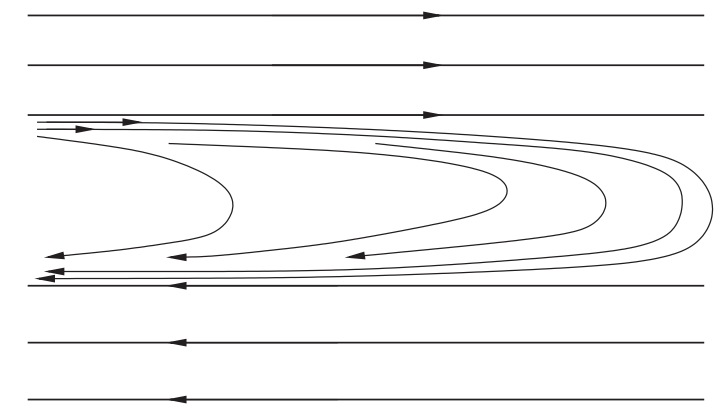

FIGURE 1. Example of a free map with three fundamental regions.

In fact the condition $a<-1$ corresponds to the set of parameter values for which $H_{a}$ do not have fixed points. Hence, the Brouwer translation theorem guarantees that, for each $x \in \mathbb{R}^{2}$, there exists an open, connected and invariant neighbourhood $V$ of $x$ such that $\left.H_{a}\right|_{V}$ is topologically conjugated to a translation of the plane (see, for example, [6]). However, this does not imply that the map $H_{a}$ is topologically conjugated to a translation, that is the neighbourhood $V$ is not necessarily equal to $\mathbb{R}^{2}$ (see, for example, Figure 1 or [7, Figure 2]). Here we prove that, for this set of parameters, $H_{a}$ is globally topologically conjugated to a translation in the conservative setting, therefore these maps are dynamically trivial and points cannot diverge to infinity in different ways as happens in the example given in Figure 1.

Without using Brouwer's theorem, in [4] Devaney and Nitecki proved directly that the non-wandering set $\Omega\left(H_{a}\right)(a<-1)$ of these maps is empty. This result implies that dynamically the map is simple (that is, there are no periodic points or general recurrences) but does not mean that the map is (conjugated to) a translation. Our goal is to prove that actually these maps are conjugated to translations, by constructing an area-preserving conjugacy (Sections 3 and 4) or, as an alternative approach, showing that these maps have only one fundamental region (Section 5).

In the other direction, in [4] Devaney and Nitecki proved that, for sufficiently large $a, \Omega\left(H_{a}\right)$ is a hyperbolic horseshoe. Moreover, in [3] Devaney proved that, for $a>-1$, the map $H_{a}$ has homoclinic points (not necessarily transverse homoclinic points but of the form $(x, x)$, which are called first symmetric homoclinic points) and, as a consequence of this fact, that $H_{a}$ has infinitely many periodic points. Also in [3] it is conjectured that these first symmetric homoclinic points are transverse homoclinic points. Several authors proved partial results supporting this conjecture (see [8] and the references therein). On the other hand, if one of these Hénon maps exhibits a homoclinic tangency then, as a consequence of a result of Duarte [5], it can be approximated (in the area-preserving setting) by maps with 'wild' hyperbolic sets having persistent homoclinic tangencies (leading to the phenomena of abundance of elliptic isles). Finally, the existence of the first symmetric transverse homoclinic points was used in [9] to prove that, in the real-analytic context, the centralizer of $H_{a}$ is trivial. 
Let us give an outline of the proof of Theorem 1. We start by constructing a fundamental domain of $H, \mathcal{D}$, whose saturate fills $\mathbb{R}^{2}$ (Lemma 3.1). We consider a fundamental domain $\mathcal{D}_{c}$ for the translation $T$ and split $\mathcal{D}$ and $\mathcal{D}_{c}$ into countable bounded pieces. Then we construct local diffeomorphisms, whose domains cover $\mathcal{D}$, which conjugate the restrictions of $H$ and $T$ to each piece such that each one preserves the area of its domain. Next, using the Dacorogna-Moser theorem (Theorem 4.1), we get an area-preserving homeomorphism from $\mathcal{D}$ to $\mathcal{D}_{c}$. Finally, using Lemma 3.1 and the fact that $H$ is area-preserving, we extend these maps conservatively to $\mathbb{R}^{2}$ to obtain the area-preserving homeomorphism $\Psi$.

This conservative topological conjugacy $\Psi$ trivializes $H$, which is very useful if one aims to build perturbations and remain in the area-preserving setting.

We observe that the arguments we develop to prove Lemma 3.1 may be used to prove that $H$ has only a fundamental region (Definition 5.1). Hence, by using the fact, proved by Andrea [1], that maps with only one fundamental region are topologically conjugated to $T$, we obtain another proof that $H$ and $T$ are topologically conjugated. However, this does not guarantee that there exists an area-preserving topological conjugacy.

\section{Some preliminary lemmas}

DEFINITION 2.1. We consider the map $H_{a, b}$ with parameters $a, b \in \mathbb{R}$ :

$$
\begin{gathered}
H_{a, b}: \mathbb{R}^{2} \longrightarrow \mathbb{R}^{2} \\
(x, y) \longrightarrow\left(a-x^{2}-b y, x\right) .
\end{gathered}
$$

This is the so-called Hénon map. When we consider $|b|=1$, then for all $p \in \mathbb{R}^{2}$ we have $\left|\operatorname{det}\left(D H_{a, 1}\right)_{p}\right|=1$, so the map preserves the area and that is why we call it the conservative Hénon map. If $b=1$, then, since $\operatorname{det}\left(D H_{a, 1}\right)=1, H_{a, 1}$ preserves also the orientation. Depending on the parameter $a \in \mathbb{R}, H_{a, 1}$ may, or may not, have fixed points. In fact, it is easy to see that $\operatorname{Fix}\left(H_{a, 1}\right)=\emptyset$ if and only if $a<-1$.

In what follows we deduce some properties of $H_{a, 1}$, which we will denote by $H$ and we consider $a<-1$. It is clear that $H$ is a homeomorphism with inverse

$$
H_{a, 1}^{-1}((x, y))=\left(y, a-y^{2}-x\right) .
$$

REMARK 2.1. Let $S((x, y))=(y, x)$, hence

$$
\begin{aligned}
S\left(H^{-1}(S(x, y))\right) & =S\left(H^{-1}(y, x)\right)=S\left(x, a-x^{2}-y\right) \\
& =\left(a-x^{2}-y, x\right)=H(x, y) .
\end{aligned}
$$

Therefore, $H$ is topologically conjugate to its inverse by using the symmetry on the line $y=x$, say $H \circ S=S \circ H^{-1}$. In this case the topological conjugacy is equal to its inverse, say $S=S^{-1}$. We conclude that the action of the map $H^{-1}$ may be seen as the reflection on the line $y=x$ of the action of $H$ itself. 
We shall deduce some elementary properties of the dynamics of the conservative Hénon map. Let us define

$$
\begin{aligned}
\mathrm{I} & =\{(x, y): x, y \geq 0\}, \\
\mathrm{II} & =\{(x, y): x \leq 0, y \geq 0\}, \\
\mathrm{III} & =\{(x, y): x, y \leq 0\} \\
\mathrm{IV} & =\{(x, y): x \geq 0, y \leq 0\} .
\end{aligned}
$$

The subset of III defined by $\left\{(x, y) \in \mathbb{R}^{2}: x \leq y\right\}$ will be denoted by $D^{+}$, and we finally denote $S\left(D^{+}\right)$by $D^{-}$.

\section{LEMMA 2.1. One has:}

(1) $H\left(D^{+}\right) \subseteq D^{+}$and $H^{n}\left(D^{+}\right) \subseteq \operatorname{int}\left(D^{+}\right)$, for all $n \geq 2$;

(2) $H^{-1}\left(D^{-}\right) \subseteq D^{-}$and $H^{-n}\left(D^{-}\right) \subseteq \operatorname{int}\left(D^{-}\right)$, for all $n \geq 2$.

PROOF. First, using Remark 2.1 and the definition of $D^{-}$, note that

$$
H^{-1}\left(D^{-}\right)=H^{-1} S\left(\left(D^{+}\right)\right)=S \circ H\left(D^{+}\right) .
$$

Therefore (2) follows from (1).

Now fix $(x, y) \in D^{+}$and let $\left(x_{1}, y_{1}\right)=H(x, y)=\left(a-x^{2}-y, x\right)$. It is clear that $y_{1}=x \leq 0$ and, since $a-x^{2}-x \leq x$, for all $x \in \mathbb{R}$, we obtain

$$
x_{1}=a-x^{2}-y \leq a-x^{2}-x \leq x=y_{1} .
$$

Therefore $\left(x_{1}, y_{1}\right) \in D^{+}$, which implies that $H\left(D^{+}\right) \subseteq D^{+}$. Finally, to prove that $H^{n}\left(D^{+}\right) \subseteq \operatorname{int}\left(D^{+}\right)$, for all $n \geq 2$, we first note that $(0,0)$ is the unique point of $D^{+}$ whose image belongs to the boundary of $D^{+}$.

Since

$$
H^{2}((0,0))=H((a, 0))=\left(a-a^{2}, a\right) \in \operatorname{int}\left(D^{+}\right),
$$

we conclude that $H^{2}\left(D^{+}\right) \subseteq \operatorname{int}\left(D^{+}\right)$and so

$$
H^{n}\left(D^{+}\right)=H^{n-2}\left(H^{2}\left(D^{+}\right)\right) \subseteq H^{n-2}\left(\operatorname{int}\left(D^{+}\right)\right) \subseteq \operatorname{int}\left(D^{+}\right) \text {for all } n \geq 2 .
$$

LEMMA 2.2. Given $(x, y) \in \mathbb{R}^{2}$ we have:

(1) if $(x, y) \in \mathrm{I}$, then $H((x, y)) \in \mathrm{II}$;

(2) if $(x, y) \in \mathrm{II}$, then $H((x, y)) \in D^{+}$;

(3) if $(x, y) \in \mathrm{IV}$, then $H((x, y)) \in \mathrm{I}$ or $H((x, y)) \in \mathrm{II}$;

(4) if $(x, y) \in \mathrm{III}$, then $H((x, y)) \in \mathrm{III}$ or $H((x, y)) \in \mathrm{IV}$;

(5) if $H^{n}((x, y)) \in \mathrm{III}$, for all $n \in \mathbb{N}_{0}$, then there exists $k \in \mathbb{N}_{0}$ such that $H^{k}((x, y)) \in D^{+}$. 

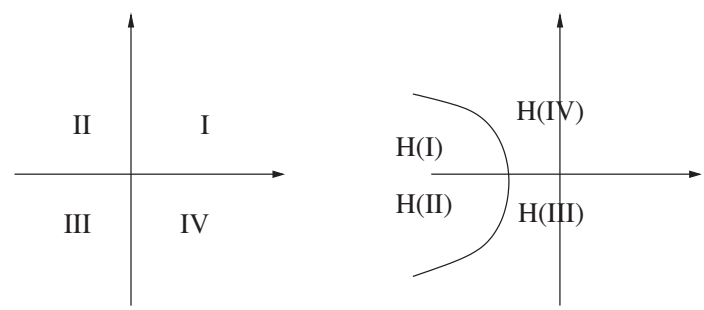

FIGURE 2. Images of the four regions.

Proof. (1) If $(x, y) \in \mathrm{I}$ then, since $H((x, y))=\left(a-x^{2}-y, x\right), a-x^{2}-y \leq$ $a<0$ and $x \geq 0$, we obtain $H((x, y)) \in$ II.

(2) If $(x, y) \in \mathrm{II}$, then $x \leq 0$ and $y \geq 0$. Since $H((x, y))=\left(a-x^{2}-y, x\right)$ it remains to prove that $a-x^{2}-y \leq x$. Since $a<-1$ for all $x \in \mathbb{R}$ it follows that $a-x^{2}-2 x \leq 0$, therefore $a-x^{2}-x \leq x$ and, since $-y \leq-x$, we conclude that $a-x^{2}-y \leq a-x^{2}-x \leq x$. So $H((x, y)) \in D^{+}$.

(3) If $(x, y) \in \mathrm{IV}$, then since $H((x, y))=\left(a-x^{2}-y, x\right)$ and $x \geq 0$ it is clear that $H((x, y))$ belongs to I or to II.

(4) If $(x, y) \in \mathrm{III}$, then, since $H((x, y))=\left(a-x^{2}-y, x\right)$, having $H((x, y))$ in III or in IV is equivalent to $x \leq 0$, which is obvious.

(5) Consider $\left(x_{0}, y_{0}\right) \in$ III such that $H^{n}\left(\left(x_{0}, y_{0}\right)\right) \in$ III, for all $n \in \mathbb{N}$. Assume that $H^{n}\left(\left(x_{0}, y_{0}\right)\right) \notin D^{+}$, for all $n \in \mathbb{N}$. Denote $\left(x_{n}, y_{n}\right)=H^{n}\left(\left(x_{0}, y_{0}\right)\right)$ and we have

$$
0 \geq x_{n} \geq y_{n}=x_{n-1} \quad \text { for all } n \in \mathbb{N} \text {. }
$$

So, $\left(x_{n}\right)_{n \in \mathbb{N}}$ is an increasing bounded sequence. Therefore it converges to $p \in \mathbb{R}_{0}^{+}$. Since $y_{n}=x_{n-1}$ it follows that $\left(y_{n}\right)_{n \in \mathbb{N}} \underset{n \rightarrow+\infty}{\longrightarrow} p$. So, $H^{n}\left(\left(x_{0}, y_{0}\right)\right) \underset{n \rightarrow+\infty}{\longrightarrow}(p, p)$ and so $(p, p)$ is a fixed point for $H$, which is a contradiction.

Lemmas 2.1 and 2.2 allow us to conclude that for all $(x, y) \in \mathbb{R}^{2}$ there exists $n_{0} \in \mathbb{N}$, depending on $(x, y)$, such that $H^{n}((x, y)) \in \operatorname{int}\left(D^{+}\right)$, for all $n \geq n_{0}$. Analogously we conclude that exists $n_{1} \in \mathbb{N}$ such that $H^{-n}((x, y)) \in \operatorname{int}\left(D^{-}\right)$, for all $n \geq n_{1}$. It follows that we just need to know the dynamics of $H$ inside $D^{+}$(respectively the dynamics of $H^{-1}$ inside $D^{-}$). Figure 2 illustrates the image by $H$ of the four regions.

LeMma 2.3. Let $\left(x_{0}, y_{0}\right) \in D^{+}$and define $\left(x_{n}, y_{n}\right)=H^{n}\left(\left(x_{0}, y_{0}\right)\right)$. Then there exists $n_{0} \in \mathbb{N}$, depending on $\left(x_{0}, y_{0}\right)$, such that for all $n \in \mathbb{N}$ with $n \geq n_{0}$ we have:

(1) $x_{n+1} \leq x_{n}+a$;

(2) $y_{n+1} \leq y_{n}$ e $y_{n+2} \leq y_{n}+a$.

PRoOF. Given $\left(x_{1}, y_{1}\right)=\left(a-x_{0}^{2}-y_{0}, x_{0}\right)$ we have that $y_{1}=x_{0} \leq y_{0}$ and

$$
x_{1}=a-x_{0}^{2}-y_{0} \leq a-x_{0}^{2}-x_{0} \leq x_{0},
$$


so the sequences $\left(x_{n}\right)_{n \in \mathbb{N}}$ and $\left(y_{n}\right)_{n \in \mathbb{N}}$ are decreasing. Note that, for all $(x, y) \in D^{+}$, there exists $n_{0} \in \mathbb{N}$ such that $x_{n_{0}}<-2$ because otherwise it implies the existence of a fixed point. Since for $x<-2$ we have

$$
x_{n+1}=a-x_{n}^{2}-y_{n} \leq a-x_{n}^{2}-x_{n} \leq x_{n}+a,
$$

inequality (1) is proved.

To prove (2) we note that the first inequality follows from $\left(y_{n}\right)_{n \in \mathbb{N}}$ being decreasing and the second inequality follows directly from the fact that $y_{n+2}=x_{n+1}$ $\leq x_{n}+a \leq y_{n}+a$.

Lemma 2.3 tell us that, under only two iterates and since $a<-1$, points move down by a distance of at least one and to the left by a distance larger than two. So this result together with Lemmas 2.1 and 2.2 give us a very good description of how the orbit of a point tends to infinity.

\section{Construction of the topological conjugacy between the $T$ and $H$}

First we define the fundamental domain $\mathcal{D}$. Let $\lambda=\{(x, y): y=x\}$ and $H(\lambda)$ be its image by $H$. Let $\mathcal{D}$ be the connected, unbounded region of the plane delimited by $\lambda$ and $H(\lambda)$. We shall see first that the saturate of $\mathcal{D}$ fills all of the plane.

LEMMA 3.1. We have $\bigcup_{n \in \mathbb{Z}} H^{n}(\mathcal{D})=\mathbb{R}^{2}$.

Proof. Note that it is sufficient to prove that, given $(x, y)$ with $x \leq y$, there exists $n \in \mathbb{N}_{0}$ such that $H^{-n}((x, y)) \in \mathcal{D}$, because for the region $x \geq y$ we use the fact that $H^{-1}=S \circ H \circ S$. Since $H^{2}(\lambda)$ is contained in the region III it follows that, for $(x, y) \in \mathrm{II} \backslash \mathcal{D}$, we have that $H^{-1}((x, y)) \in \mathcal{D}$. It remains to consider points $(x, y) \in$ $D^{+} \backslash \mathcal{D}$. So, $H^{-1}((x, y))$ belongs to II or to III. Note that, if $H^{-1}((x, y)) \in$ II or more generally there exists $n \in \mathbb{N}$ such that $H^{-n}((x, y)) \in \mathrm{II}$, then $H^{-1}((x, y)) \in \mathcal{D}$ or $H^{-2}((x, y)) \in \mathcal{D}$. Then, we just have to consider the case when $(x, y) \in D^{+}$ and $H^{-n}((x, y)) \in \mathrm{III}$, for all $n \in \mathbb{N}$. Suppose, by contradiction, that $H^{-n}((x, y)) \notin$ $\mathcal{D}$, for all $n \in \mathbb{N}$; then, since $(x, y) \in D^{+} \backslash \mathcal{D}$ we obtain $x<a-y^{2}-y$ and so $H^{-1}((x, y)) \in D^{+}$. We obtain a sequence in $D^{+},\left(x_{-n}, y_{-n}\right)=H^{-n}((x, y)), n \in \mathbb{N}$, such that $x_{-n} \geq x_{-n+1}$ and $y_{-n} \geq y_{-n+1}$, for all $n \in \mathbb{N}$. Therefore $\left(\left(x_{-n}, y_{-n}\right)\right)_{n \in \mathbb{N}}$ converges to $\left(x_{0}, y_{0}\right)$ which is a fixed point, and we obtain a contradiction.

REMARK 3.1. Clearly int $(\mathcal{D}) \cap H^{n}(\operatorname{int}(\mathcal{D}))=\emptyset$, for all $n \in \mathbb{Z} \backslash\{0\}$.

Now we shall construct the topological conjugacy, that is, a homeomorphism $\Phi: \mathbb{R}^{2} \longrightarrow \mathbb{R}^{2}$ such that $\Phi \circ H=T \circ \Phi$, where $T: \mathbb{R}^{2} \longrightarrow \mathbb{R}^{2}$ is the translation $T((x, y))=(x+1, y)$. Let $\lambda_{c}$ denote the line $x=0$ and

$$
\mathcal{D}_{c}=\left\{(x, y) \in \mathbb{R}^{2}: 0 \leq x \leq 1\right\},
$$



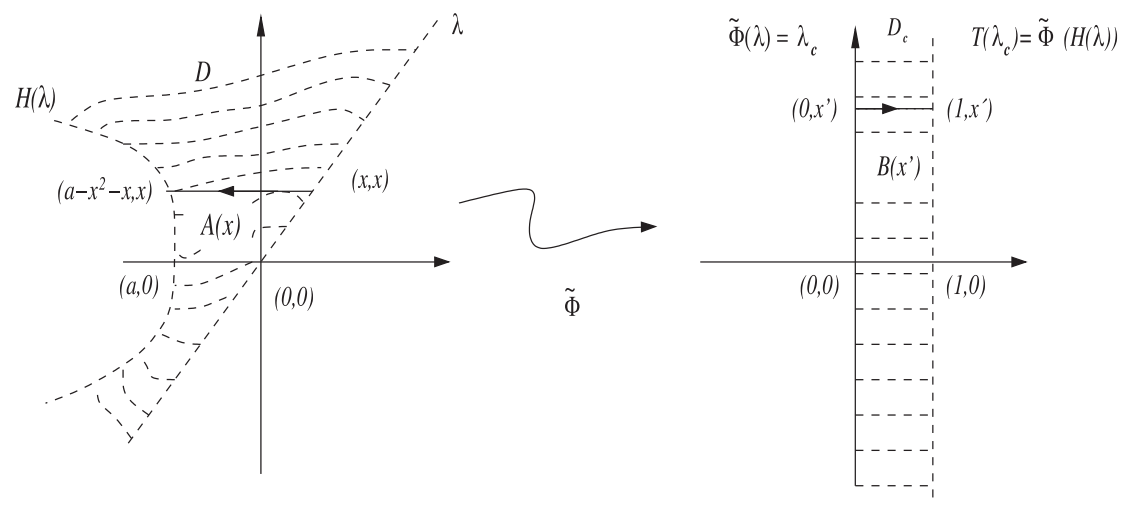

FIGURE 3. Construction of the topological conjugacy.

the idea is to construct first a homeomorphism $\widetilde{\Phi}: \mathcal{D} \longrightarrow \mathcal{D}_{c}$ such that $\widetilde{\Phi}(\lambda)=\lambda_{c}$ and $\widetilde{\Phi}(H(x))=T(\widetilde{\Phi}(x))$, for all $x \in \lambda$, and then extend it to $\mathbb{R}^{2}$.

Denote by $[a, b]$ the oriented segment from the point $a$ through the point $b$, and, for $x \neq 0$, let $\mathcal{A}(x)$ be the region bounded by the three segments $[(x, x),(0,0)]$, $\left[\left(a-x^{2}-x, x\right),(x, x)\right]$ and $[(0,0),(a, 0)]$ and by the curve $H([(0,0),(x, x)])$; for $x^{\prime} \neq 0$ let $\mathcal{B}\left(x^{\prime}\right)$ be the region delimited by the quadrilateral with vertices $(0,0),(1,0)$, $\left(1, x^{\prime}\right)$ and $\left(0, x^{\prime}\right)$.

We want a diffeomorphism $\widetilde{\Phi}$ such that, for all $x \neq 0$ :

(1) $\widetilde{\Phi}([(0,0),(x, x)])=\left[(0,0),\left(0, x^{\prime}\right)\right]$;

(2) $\widetilde{\Phi} \circ H((x, x))=T \circ \widetilde{\Phi}((x, x))$; and

(3) $\widetilde{\Phi}(\mathcal{A}(x))=\mathcal{B}\left(x^{\prime}\right)$;

where $x^{\prime}$ depends on $x$ and will be chosen such that $\mu(\mathcal{A}(x))=\mu\left(\mathcal{B}\left(x^{\prime}\right)\right)$, where $\mu$ denotes the Lebesgue measure; see Figure 3.

3.1. Construction of $\tilde{\boldsymbol{\Phi}}$ We consider the parameterize segment:

$$
(1-t)(x, x)+t\left(a-x^{2}-x, x\right), \quad t \in[0,1]
$$

and send this segment, by the map $\widetilde{\Phi}$, into the segment

$$
(1-t)\left(0, x^{\prime}\right)+t\left(1, x^{\prime}\right), \quad t \in[0,1]
$$

where $x^{\prime}$ will be defined below. We have that

$$
\mu(\mathcal{A}(x))=\int_{0}^{|x|} t-\left(a-t^{2}-t\right) d t=-a|x|+\frac{|x|^{3}}{3}+x^{2}, \quad x \in \mathbb{R} \backslash\{0\} .
$$

So we take: 
(1) $x^{\prime}:=-a x+\left(x^{3} / 3\right)+x^{2}$, if $x>0$; and

(2) $\quad x^{\prime}:=-\left(-a|x|+\left(|x|^{3} / 3\right)+x^{2}\right)=-a x+\left(x^{3} / 3\right)-x^{2}$, otherwise.

Therefore, if $x \geq 0$, we define

$$
\widetilde{\Phi}\left(\left((1-t) x+t\left(a-x^{2}-x\right), x\right)\right)=\left(t, x^{\prime}\right)=\left(t,-a x+\frac{x^{3}}{3}+x^{2}\right),
$$

and, if $x \leq 0$, we define

$$
\widetilde{\Phi}\left(\left((1-t) x+t\left(a-x^{2}-x\right), x\right)\right)=\left(t, x^{\prime}\right)=\left(t,-a x+\frac{x^{3}}{3}-x^{2}\right),
$$

Now, let $(x, y) \in \operatorname{int}(\mathcal{A}(x))$ and assume that $y \geq 0$; this point belongs to $\left[(y, y),\left(a-y^{2}-y, y\right)\right]$, so there exists one and only one $t \in[0,1]$ such that

$$
(x, y)=(1-t)(y, y)+t\left(a-y^{2}-y, y\right) .
$$

Hence, we obtain

$$
x=(1-t) y+t\left(a-y^{2}-y\right) \Leftrightarrow x-y=t\left(a-y^{2}-2 y\right) \Leftrightarrow t=\frac{x-y}{a-y^{2}-2 y},
$$

and since $\widetilde{\Phi}((y, y))=\left(0, y^{\prime}\right)$ and $\widetilde{\Phi}\left(\left(a-y^{2}-y, y\right)\right)=\left(1, y^{\prime}\right)$ we can define

$$
\widetilde{\Phi}((x, y))=(1-t)\left(0, y^{\prime}\right)+t\left(1, y^{\prime}\right)=\left(t, y^{\prime}\right)=\left(\frac{x-y}{a-y^{2}-2 y}, y^{\prime}\right) \text {. }
$$

If $y \leq 0$ we can argue exactly in the same way and define

$$
\widetilde{\Phi}((x, y))=(1-t)\left(0, y^{\prime}\right)+t\left(1, y^{\prime}\right)=\left(t, y^{\prime}\right)=\left(\frac{x-y}{a-y^{2}-2 y}, y^{\prime}\right) .
$$

Using the previous definition of $y^{\prime}$ we conclude that the homeomorphism $\widetilde{\Phi}: \mathcal{D} \longrightarrow \mathcal{D}_{c}$ is defined by

$$
\widetilde{\Phi}((x, y))= \begin{cases}\left(\frac{x-y}{a-y^{2}-2 y},-a y+\frac{y^{3}}{3}+y^{2}\right) & \text { if } y \geq 0, \\ \left(\frac{x-y}{a-y^{2}-2 y},-a y+\frac{y^{3}}{3}-y^{2}\right) & \text { if } y \leq 0 .\end{cases}
$$

Clearly $\mu(\mathcal{A}(y))=\mu\left(\mathcal{B}\left(y^{\prime}\right)\right)$. Note that, by construction, for all $p \in \lambda$ we have $\widetilde{\Phi} \circ H(p)=T \circ \widetilde{\Phi}(p)$.

3.2. Construction of $\boldsymbol{\Phi}$ In order to obtain the desired topological conjugacy $\Phi$ we extend $\widetilde{\Phi}$ to the plane in the standard way. Lemma 3.1 assures that, for each $p \in \mathbb{R}^{2}$, there exists $n_{p} \in \mathbb{Z}$ such that $H^{n_{p}}(p) \in \mathcal{D}$; therefore, we define $\Phi(p)=$ $T^{-n_{p}} \circ \widetilde{\Phi} \circ H^{n_{p}}(p)$. It is easy to prove that the map $\Phi$ is well defined, that it is a homeomorphism and that $\Phi \circ H=T \circ \Phi$. 


\section{Obtaining the area-preserving topological conjugacy}

Write $\mathcal{D}=\bigcup_{i \in \mathbb{Z}} C_{i}$, where, for each $i \in \mathbb{Z}, C_{i}=\mathcal{A}(i) \backslash \mathcal{A}(i-1)$. In order to construct the area-preserving topological conjugacy $\Psi$ we first modify $\widetilde{\Phi}$ in the interior of each bounded region $C_{i}$ in such way that the resulting map, say $\widetilde{\Psi}_{i}$, is areapreserving and coincides with $\widetilde{\Phi}$ on $\partial C_{i}$. As the maps $\widetilde{\Psi}_{i}$ and $\widetilde{\Psi}_{i+1}$ coincide on $\left[(i, i),\left(a-i^{2}-i, i\right)\right]$, for all $i \in \mathbb{Z}$, these maps define an area-preserving map on $\mathcal{D}$, say $\widetilde{\Psi}$, such that $\widetilde{\Psi}(\mathcal{D})=\mathcal{D}_{c}$; as the maps $\widetilde{\Psi}_{i}$ coincide with $\widetilde{\Phi}$ on $\lambda$ and $H(\lambda)$ one has that $\widetilde{\Psi} \circ H(p)=T \circ \widetilde{\Psi}(p)$, for all $p \in \lambda$. Finally the area-preserving topological conjugacy is obtained extending the map $\widetilde{\Psi}$ to the plane as before.

Let us recall the following theorem due to Dacorogna and Moser [2, Theorem 5] that will be used to obtain a conservative local change of coordinates that is crucial to obtain the maps $\widetilde{\Psi}_{i}$. We point out that this version of the Dacorogna-Moser theorem considers only regularity in the interior of a set $\Omega$, whereas [2, Theorem 1] requires a smooth boundary and as a consequence a smooth change of coordinates is obtained. Since we are only interested in homeomorphisms for the change of coordinates the following theorem is sufficient.

THEOREM 4.1 (Dacorogna-Moser). Let $\Omega \subset \mathbb{R}^{2}$ be an open, bounded and connected set. Let $f, g: \bar{\Omega} \rightarrow \mathbb{R}$ be positive functions of class $C^{s}$ for $s \in \mathbb{N}$ and such that $\int_{\Omega} f(y) d y=\int_{\Omega} g(y) d y$. Then there exists a diffeomorphism $\varphi: \bar{\Omega} \rightarrow \bar{\Omega}$ of class $C^{s}$ with $\varphi(x)=x$ for $x \in \partial \Omega$ and such that $\int_{U} f(y) d y=\int_{\varphi(U)} g(y) d y$ for every open set $U \subset \Omega$.

By a straightforward use of change of variables we obtain

$$
\int_{\varphi(U)} g(y) d y=\int_{U} g(\varphi(y)) \operatorname{det} D \varphi_{y} d y,
$$

but by Theorem 4.1 we know that $\int_{\Omega} f(y) d y=\int_{\Omega} g(y) d y$ so

$$
\int_{U} f(y) d y=\int_{U} g(\varphi(y)) \operatorname{det} D \varphi_{y} d y
$$

and since this works for every open set $U$ we obtain

$$
\operatorname{det} D \varphi_{y} g(\varphi(y))=f(y) .
$$

Now fix $x=1$ and consider the associated $x^{\prime}=4 / 3-a$ as in the previous section. Let $\operatorname{int}\left(\mathcal{B}\left(x^{\prime}\right)\right)=\Omega$ and we consider the functions $f, g: \bar{\Omega} \rightarrow \mathbb{R}$, defined by $f(y)=$ $\left|\operatorname{det} D \widetilde{\Phi}_{y}^{-1}\right|$ and $g=1$. We note that $f \in C^{0}(\bar{\Omega})$.

CLAIM. We claim that $\int_{\Omega} f(y) d y=\int_{\Omega} g(y) d y$. 
Proof. To see this we observe that

$$
\frac{\int_{\Omega} g(y) d y}{\int_{\Omega} f(y) d y}=\frac{\mu(\Omega)}{\int_{\Omega}\left|\operatorname{det} D \widetilde{\Phi}_{y}^{-1}\right| d y}=\frac{\mu(\Omega)}{\mu\left(\widetilde{\Phi}^{-1}(\Omega)\right)}=\frac{\mu\left(\mathcal{B}\left(x^{\prime}\right)\right)}{\mu(\mathcal{A}(x))}=1 .
$$

The last equality follows by construction and the claim is proved.

Hence we can apply Theorem 4.1 and we obtain a homeomorphism $\varphi: \bar{\Omega} \rightarrow \bar{\Omega}$ satisfying (1).

Now we define

$$
\begin{aligned}
& \widetilde{\Psi}_{1}: \mathcal{A}(1) \longrightarrow \bar{\Omega} \\
& p \longrightarrow \varphi \circ \widetilde{\Phi}(p) .
\end{aligned}
$$

To see that $\widetilde{\Psi}_{1}$ is area-preserving we compute the determinant of the Jacobian,

$$
\left|\operatorname{det} D \widetilde{\Psi}_{1}\right|=|\operatorname{det} D \varphi \cdot \operatorname{det} D \widetilde{\Phi}|=\left|\operatorname{det} D \varphi \cdot f^{-1}\right| .
$$

Now the partial differential equation (1) says that det $D \varphi \cdot f^{-1}=1$, so therefore we obtain that det $D \widetilde{\Psi}_{1}=1$, that is, $\widetilde{\Psi}_{1}$ is area-preserving.

In the same way we obtain $\widetilde{\Psi}_{-1}$.

The maps $\widetilde{\Psi}_{i}: \mathcal{A}(i) \backslash \mathcal{A}(i-1) \rightarrow \mathcal{B}\left(x_{i}^{\prime}\right) \backslash \mathcal{B}\left(x_{i-1}^{\prime}\right), \quad i \in \mathbb{Z}$, are obtained by applying recursively the previous arguments to the points $x_{i}=i$ and the corresponding $x_{i}^{\prime}$, and to the region $\Omega=\operatorname{int}\left(\mathcal{B}\left(x_{i}^{\prime}\right) \backslash \mathcal{B}\left(x_{i-1}^{\prime}\right)\right)$, thus ending the proof of Theorem 1.

\section{Existence of a topological conjugacy via fundamental regions}

Let $f: \mathbb{R}^{2} \longrightarrow \mathbb{R}^{2}$ be a free map of the plane, say a fixed point free homeomorphism of the plane which preserves the orientation. We define an equivalence relation by saying that $x$ is related with $y$, denoted by $x \sim y$, if there exists a compact arc $\gamma$ from $x$ through $y$ such that, given any compact $K \subseteq \mathbb{R}^{2}$, there exists $n_{0} \in \mathbb{N}$ such that for any $n>n_{0}$ and $n<-n_{0}$ we have $H^{n}(\gamma) \cap K=\emptyset$.

DEFINITION 5.1. Each element of $\mathbb{R}^{2} / \sim$ is called a fundamental region.

Fundamental regions are pairwise disjoint and invariant by topological conjugacies. A free map of the plane may even have infinitely many fundamental regions; however, it is straightforward to see that a translation has only one. In fact, Andrea (see [1]) proved the following result.

THEOREM 5.1. A free map of the plane is topologically conjugated to a translation if and only if it has just one fundamental region.

Hence to prove that the conservative Hénon map is topologically conjugated to a translation it is sufficient to show that this map has only one fundamental region. 


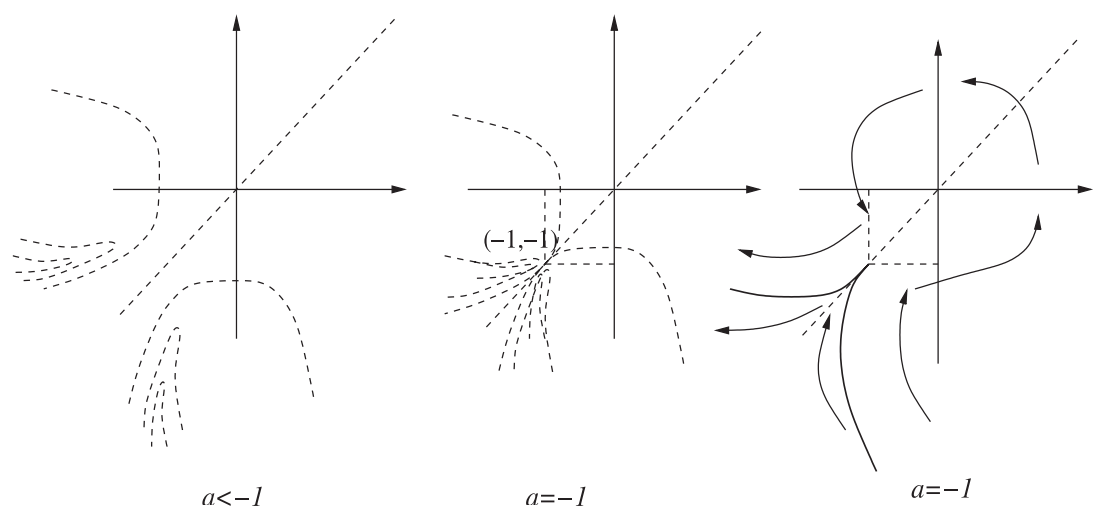

FIGURE 4. The bifurcation parameter $a=-1$.

Proposition 5.2. The map $H_{a}, a<-1$, has only one fundamental region.

PROOF. Let $x, y \in \mathbb{R}^{2}$, let $\gamma$ be a compact segment from $x$ through $y$ and let $K \subseteq \mathbb{R}^{2}$ be any compact set. Clearly $K \subseteq Q_{K}$ for some square $Q_{K}$ centred in the origin. Lemmas 2.1 to 2.3 are sufficient to conclude that there exists $n_{k} \in \mathbb{N}$ such that, for all $n>n_{k}$, we have $H^{n}(\gamma) \cap Q_{K}=\emptyset$. In fact, if we fix $z \in \gamma$, there exists $n_{z} \in \mathbb{N}$ such that $H^{n_{z}}(z) \notin Q_{K}, H^{n_{z}}(z) \in \operatorname{int}\left(D^{+}\right)$and the first coordinate of $H^{n_{z}}(z)$ is smaller than -2 . Therefore, for all $n>n_{z}$ we have $H^{n}(z) \notin Q_{K}$ and by continuity of $n_{z}$ it is true also for some open neighbourhood of $z, V_{z}$, sufficiently small. Let $\left\{V_{z}\right\}_{z \in \gamma}$ be an open covering of $\gamma$; since $\gamma$ is compact $\left\{V_{z}\right\}_{z \in \gamma}$ admits a finite sub covering $\left\{V_{z_{1}}, \ldots, V_{z_{s}}\right\}$ and, as above, to each $z_{i}$ we associate $n_{z_{i}}$ such that $H^{n_{z_{i}}}\left(V_{z_{i}}\right) \cap Q_{K}=\emptyset, H^{n_{z_{i}}}\left(V_{z_{i}}\right) \subseteq D^{+}$and the first coordinate of any point of $H^{n_{z_{i}}}\left(V_{z_{i}}\right)$ is less than -2 . Now, we just choose $n_{0}=\max \left\{n_{z_{1}}, \ldots, n_{z_{s}}\right\}$ and we have that, for $n>n_{0}, H^{n}(\gamma) \cap K=\emptyset$. By an analogous procedure we prove that $H^{-n}(\gamma) \cap K=\emptyset$, by using the map $S$ in $D^{-}$.

We note that this argument does not assure the existence of an area-preserving topological conjugacy.

\section{Final remarks}

In the case of the conservative Hénon map with $a=-1$, our map is no longer a free map, because it has a fixed point. Therefore, it is not conjugate to a translation. Nevertheless, the dynamics of this new map seems to be very similar to the case when $a<-1$, see Figure 4. After the bifurcation, say for $a>-1$, the dynamics have rich properties, namely horseshoes and elliptical islands.

For the reversing-orientation conservative Hénon map without fixed points, that is when $b=-1$ and $a<0$ in Definition 2.1, it is straightforward to prove that 
$H \circ R=R \circ H^{-1}$, where $H=H_{a,-1}$ and $R(x, y)=(-y,-x)$. Therefore, considering the region $\mathcal{D}$ bounded by the curves $\lambda=\left\{(x, y) \in \mathbb{R}^{2}: y=-x\right\}$ and $H(\lambda)$, we believe that, applying the same arguments, it is possible to prove that $\mathcal{D}$ is a fundamental domain and that there exists an area-preserving topological conjugacy between $H$ and the map $Z(x, y)=(x+1,-y)$.

\section{References}

[1] S. Andrea, 'On Homeomorphisms on the plane which have no fixed points', Abh. Math. Semin. Univ. Hamburg 30 (1967), 61-74.

[2] B. Dacorogna and J. Moser, 'On a partial differential equation involving the Jacobian determinant', Ann. Inst. H. Poincaré Anal. Non. Linéaire 7 (1990), 1-26.

[3] R. Devaney, 'Homoclinic bifurcations and the area-conserving Hénon mapping', J. Differential Equations 51 (1984), 254-266.

[4] R. Devaney and Z. Nitecki, 'Shift automorphisms in the Hénon mapping', Comm. Math. Phys. 67 (1979), 137-146.

[5] P. Duarte, 'Abundance of elliptic isles at conservative bifurcations', Dynam. Stability Systems 14(4) (1999), 339-356.

[6] J. Franks, 'A new proof of the Brouwer plane translation theorem', Ergod. Th. \& Dynam. Sys. 12 (1992), 217-226.

[7] L. Guillou, 'Brouwer's plane translation theorem and generalizations of the Poincaré-Birkhoff theorem', Topology 22 (1994), 331-351.

[8] U. Kirchgraber and D. Stoffer, 'Transversal homoclinic points of the Hénon map', Ann. Mat. Pura Appl. 185 (2006), S187-S204.

[9] J. Rocha, 'Centralizers and entropy', Bol. Soc. Brasil Mat. (N.S.) 25 (1994), 213-222.

\section{CMUP}

Rua do Campo Alegre

687, 4169-007 Porto

Portugal

e-mail: bessa@fc.up.pt
DMP-FCUP

Rua do Campo Alegre 687, 4169-007 Porto

Portugal

e-mail: jrocha@fc.up.pt 